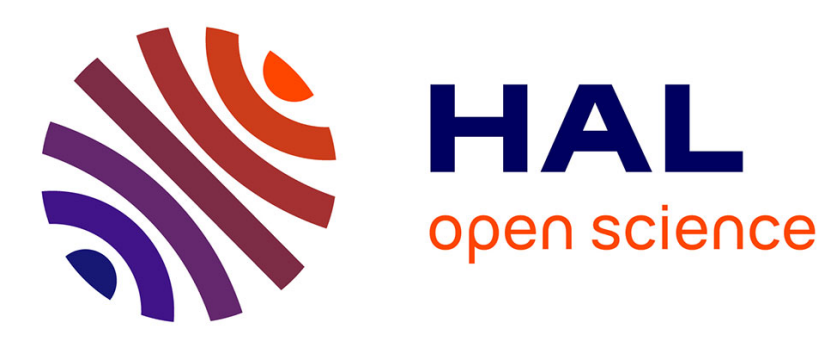

\title{
Energy Efficiency Analysis of JT-CoMP Scheme in Macro/Femto Cellular Networks
}

\author{
Yanqiao Hou, Lynda Zitoune, Véronique Vèque
}

\section{To cite this version:}

Yanqiao Hou, Lynda Zitoune, Véronique Vèque. Energy Efficiency Analysis of JT-CoMP Scheme in Macro/Femto Cellular Networks. GLOBECOM 2019 - 2019 IEEE Global Communications Conference, Dec 2019, Waikoloa, United States. 10.1109/GLOBECOM38437.2019.9013282 . hal-02943611

\section{HAL Id: hal-02943611 \\ https://hal-centralesupelec.archives-ouvertes.fr/hal-02943611}

Submitted on 20 Sep 2020

HAL is a multi-disciplinary open access archive for the deposit and dissemination of scientific research documents, whether they are published or not. The documents may come from teaching and research institutions in France or abroad, or from public or private research centers.
L'archive ouverte pluridisciplinaire HAL, est destinée au dépôt et à la diffusion de documents scientifiques de niveau recherche, publiés ou non, émanant des établissements d'enseignement et de recherche français ou étrangers, des laboratoires publics ou privés. 


\title{
Energy Efficiency Analysis of JT-CoMP Scheme in Macro/Femto Cellular Networks
}

\author{
Yanqiao Hou*, Lynda Zitoune ${ }^{\dagger *}$, Véronique Vèque* \\ * Signals and Systems Laboratory (L2S) \\ CentraleSupelec-CNRS-Paris-Sud University, Paris-Saclay University \\ Email: \{yanqiao.hou, veronique.veque\}@12s.centralesupelec.fr \\ $\dagger$ Systems Engineering Department, Esiee-Paris \\ Email: lynda.zitoune@esiee.fr
}

\begin{abstract}
G Wireless Networks are expected to increase substantially data rates and quality of service the users will experience, with a similar or a lower power consumption as todays 4G networks. The Joint Transmission Coordinated MultiPoint (JTCoMP) is a promising scheme to enhance throughput by reducing the interference, especially for cell-edge users. However, some additional energy for hardware circuit and resource information is consumed by this technology. Meanwhile, the performance evaluation of energy efficiency (EE) in dense networks with JTCoMP approach becomes a hard task in terms of time expense to conduct simulations. To evaluate the EE metric in cellular networks with JT-CoMP scheme and to capture the major factors involved in the energy consumption process, representative and accurate models are needed. In this paper, we develop a tractable and efficient model of EE based on spatial fluid modeling when JT-CoMP is applied. Simulations results show that EE is improved with the raise of the number of coordinated BSs in case of a constant backhauling power cost. Similar EE improvement is also observed in case of variable backhauling power cost, while adding a new coordinated BS. Furthermore, the EE is significantly enhanced in femto cellular networks compared to macro cellular ones, making thereby, JT-CoMP scheme more effective in small cells.
\end{abstract}

\section{INTRODUCTION}

The energy efficiency, as one of the key performance indicators in 5G network, has been attracted much interest in the recent years. International Telecommunication Union (ITU) sets the design target of energy efficiency for 5G network to be 100 -fold than currently available $4 \mathrm{G}$ system, along with more consistent transmission data rate of $100 \mathrm{Mbps}$ and $1 \mathrm{~ms}$ of latency [1], [2]. Additionally, the energy efficiency (denoted EE throughout this paper) is also drawn much attention from the perspectives of reducing environmental pollution and saving operational cost [3].

Most of advanced techniques launched for Advanced-4G are exploited to improve EE for future 5G networks, such as Cloud Radio Access Networks [4], Massive Multiple-Input Multiple-Output (MIMO) [5], [6], relay transmission [7] and the On-Off switching policy of base stations (BSs) [8]. Joint Transmission Coordinated Multipoint (JT-CoMP) is another promising technology to improve the network bit rates and fulfill upcoming communication demands [9], especially for the cell-edge user equipments (UEs). In JT-CoMP technique, information is transmitted to a UE simultaneously from different coordinated BSs in order to improve the received signal quality and strength. It enhances spectral efficiency where destructive interference is turned to constructive one. However, this technique in practice also brings additional energy cost for transmitting backhauling information. Therefore, it is interesting to investigate the EE gain and to analyse the power consumption, mainly the backhauling power cost due to the coordination. As a matter of fact, we need a tractable model to benchmark the EE variation depending on the network parameters as its size and its type (macro, femto), the number of coordinated BSs, and the path-loss exponent as it reflects the network environment.

A mathematical framework, called spatial fluid modeling, has been proposed in [10] to evaluate the network performance of 3G cellular networks, like SINR and outage probability through analytical expressions. Subsequently, in [11], the authors use this mathematical framework to study the SINR enhancement of JT-CoMP depending on the number of the coordinated BSs and other network-related parameters in dense areas. However, the EE metric is not discussed in this paper. More recently, a tractable EE model based on fluid modeling framework in [12] has been proposed to investigate the network EE for large and dense networks. In all these research activities, the obtained results show that the fluid modeling is effective to analyze large and/or dense networks, as it reduces considerably the analysis complexity and provides a macroscopic evaluation of the performance metrics, faithful to the results obtained using Monte Carlo (MC) simulations.

Naturally, we combine here our previous work [12] and [11], to develop an EE model in the case of JT-CoMP. We first extend the model in [11] to compute the total data rate over a network area. Then we derive the closed-form expression of EE for the JT-CoMP downlink transmission, which is tractable and quite simple to compute. The effectiveness and the accuracy of the underlying model are shown for both types of cellular networks, macrocells and femtocells, by comparing the results to those of MC simulations while considering several path-loss exponents and varying the number of cooperating BSs, in the case of a constant backhauling power cost. Furthermore, we investigate the EE improvement and the variation of the backhauling power cost depending on some parameters, like the network area radius and the number of coordinated BSs. 

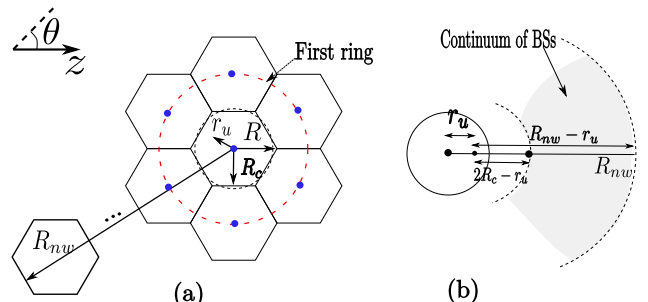

(b)

Fig. 1: (a). Hexagonal network. (b). Interference area in fluid model.

The remainder of the paper is organized as follows. First, the system model is introduced in section II, including the definition of the energy efficiency metric and the data rate computation model. Thereafter, a brief recall of the signalto-interference ratio (SIR) based on the fluid modeling is given when JT-CoMP is applied. The simulation parameters and numerical results are presented in section III. Finally, we conclude the paper in section IV.

\section{SYSTEM MODEL}

Here, we describe the EE model when JT-CoMP is used through the network, mainly within closest BSs, i.e. those belonging to the first ring as shown in Fig.1 (a). The system model presented here is quite analogous to the previous ones [12] and [11]. It concerns an OFDMA cellular network, composed of $N_{B S}$ base stations (BSs) and $N_{u}$ user equipments (UEs) randomly distributed over the network. $M_{c o}$ BSs are able to jointly transmit data in order to improve the signal quality at the UE located at the distance $r_{u}$ from its serving BS (the central cell in Fig.1 (a)). The network is supposed homogeneous, such that the transmission power $P_{t x}$ is the same for every BS.

\section{A. Energy efficiency expression}

In order to capture the energy-efficiency of that network with JT-CoMP, we use the common Eq. (1) as in [6], [13]:

$$
E E=\frac{D_{\text {area }}}{M_{c o} \times P_{e x p}^{C o M P}+\left(N_{B S}-M_{c o}\right) \times P_{e x p}} .
$$

The EE is computed as the ratio of total data rate over a network area $D_{\text {area }}$, to the total power consumption. Here, $P_{e x p}^{C o M P}$ and $P_{e x p}$ are respectively the total energy expenditure per coordinated BS and per BS.

The power consumption of a coordinated BS, $P_{\text {exp }}^{C o M P}$ in Eq. (2), is defined depending on the number of transmitting antennas $N_{a n t}$, the transmitting power $P_{t x}$ and the backhauling power cost $K_{C o M P}$. The fixed part, $P_{1}$, accounts for the direct current/alternating current (DC/AC) converter. $\Delta_{P}$ and $P_{0}$ denote some circuit power consumption.

$$
P_{\text {exp }}^{C o M P}=N_{a n t}\left(\Delta_{P} P_{t x}+P_{0}\right)+P_{1}+K_{C o M P} .
$$

$K_{C o M P}$ can be a constant for simplification, or be calculated as in [14]:

$$
K_{C o M P}=\frac{P_{b h}^{0} C_{b h}}{C_{b h}^{0}}=\alpha_{b h} C_{b h}
$$

TABLE I: Value of double linear PCM [16]

\begin{tabular}{|l|l|l|l|l|}
\hline Parameters & $P_{t x}(W)$ & $\Delta_{P}$ & $P_{0}$ & $P_{1}$ \\
\hline macro BSs & 80 & 7.25 & 244 & 255 \\
micro BSs & 6.31 & 3.14 & 35 & 34 \\
pico or femto BSs & 0.25 & 4.4 & 6.1 & 2.6 \\
\hline
\end{tabular}

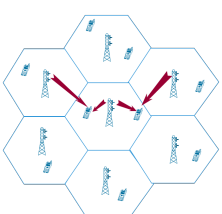

(a)

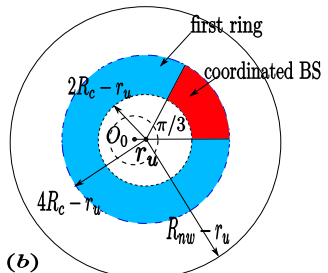

(b)
Fig. 2: JT-CoMP model (a). Hexagonal case. (b). fluid case with only one coordinated $\mathrm{BS}(n=1)$.

where $P_{b h}^{0}$ denotes the power consumed by the backhaul equipment when supporting the maximum data rate $C_{b h}^{0}$, $\alpha_{b h}=P_{b h}^{0} / C_{b h}^{0}$ is the power coefficient of backhaul equipment, and $C_{b h}$ is the backhaul traffic for every BS, i.e., the accumulated data rate of UEs served by one cooperating BS [15]. Therefore, $K_{C o M P}$ is linearly proportional to the backhaul requirement $C_{b h}$. Specially, $\alpha_{b h}=5 \times 10^{-7}$ Joules/bit for a macro BS and $\alpha_{b h}=4 \times 10^{-8}$ Joules/bit for a femto BS.

Obviously, the power consumption of a common BS, i.e., without coordination, is as the Eq. (2), without the $K_{C o M P}$ part. The numerical values of each part in Eq. (2) are listed in Table I, regarding the BSs types (macro, micro, femto) [16].

\section{B. Total data rate $D_{\text {area }}$}

The fluid paradigm assumes a continuum number of transmitters over the network [10]. Therefore, the neighbors interfering power is supposed as a continuum field, as shown by the shaded area in Fig. 1 (b), i.e., the region over rings with radii $2 R_{c}-r_{u}$ and $R_{n w}-r_{u}$, respectively, and centered at the user's position. $R_{n w}$ is the network radius. $R_{c}$ is the half distance between two BSs.

While neglecting noise, a UE $u$ at $r_{u}$ from its serving BS $b$, and receiving data from the coordinated BSs as shown in Fig. 2 (a), experiences an enhanced signal quality, $\Gamma_{u}^{C o M P}$ as

$$
\Gamma_{u}^{C o M P}=\frac{p_{u, b}+p_{u, C o M P}}{p_{u, e x t}-p_{u, C o M P}} .
$$

$p_{u, b}$ and $p_{u, C o M P}$ denote the received powers at $u$ from the serving BS and the coordinated BSs, respectively. $p_{u, e x t}$ is the sum of received interference power at $u$. We define the JT-CoMP factor $G_{u, C o M P}$ as $G_{u, C o M P}=\frac{p_{u, C o M P}}{p_{u, e x t}}$. As a consequence, the signal quality $\Gamma_{u}^{C o M P}$ in case of JT-CoMP, depends on the SIR $\Gamma_{u}$ without coordination and can be defined as follows:

$$
\Gamma_{u}^{C o M P}=\frac{\Gamma_{u}}{1-G_{u, C o M P}}+\frac{G_{u, C o M P}}{1-G_{u, C o M P}} .
$$

As shown in figure Fig.2 (b), there are $n$ BSs, such that $n=\{1, \ldots, 6\}$, in the first ring to cooperatively transmit data with the central BS. The fluid-based expression of $p_{u, C o M P}$ 
TABLE II: Simulation Parameter Value

\begin{tabular}{|l|l|}
\hline Parameters & Value \\
\hline System bandwidth B & $10 \mathrm{MHz}$ \\
Cell radius $R$ : macro, femto, resp. & $\{1000,50\} \mathrm{m}$ \\
Half distance between BSs, $R_{c}$ & $R \sqrt{3} / 2$ \\
Range of network $R_{n w}$ & $15 R_{c}$ \\
Radius of interested area $R_{a}$ & {$\left[R / 20 R_{e}\right]$} \\
Equivalent radius of one cell, $R_{e}$ & $R_{c} \sqrt{2 \sqrt{3} / \pi}$ \\
Number of antennas $N_{\text {ant }}$ & 1 \\
Number of users $N_{u}:$ macro, femto, resp. & $\{300,100\}$ \\
Path loss exponent $\eta$ & $\{2.6,3.5\}$ \\
Density of BSs $\rho_{B S}$ & $1 /\left(2 \sqrt{3} R_{c}^{2}\right)$ \\
Density of users $\rho_{u}$ & $N_{u} /\left(2 \sqrt{3} R_{c}^{2}\right)$ \\
\hline
\end{tabular}

over the cooperative area limited between $\left[2 R_{c}-r_{u}, 4 R_{c}-r_{u}\right]$ and $\left[0, \frac{n \pi}{3}\right]$, is computed using the following equation (see [11] for more details):

$$
\begin{aligned}
& p_{u, C o M P}=\int_{0}^{\frac{n \pi}{3}} \int_{2 R_{c}-r_{u}}^{4 R_{c}-r_{u}} \rho_{B S} P_{t x} A z^{-\eta} z d z d \theta \\
& =\frac{n \pi}{3} \frac{\rho_{B S} P_{t x} A}{\eta-2}\left[\left(2 R_{c}-r_{u}\right)^{2-\eta}-\left(4 R_{c}-r_{u}\right)^{2-\eta}\right],
\end{aligned}
$$

where $A$ is a constant and $\eta(>2)$ is the path-loss exponent. $\rho_{B S}=1 /\left(2 \sqrt{3} R_{c}^{2}\right)$ is the density of the BSs. Similarly, we can compute the external power $p_{u, e x t}$ as follows:

$$
\begin{aligned}
& p_{u, e x t}=\int_{0}^{2 \pi} \int_{2 R_{c}-r_{u}}^{R_{n w}-r_{u}} \rho_{B S} P_{t x} A z^{-\eta} z d z d \theta \\
& =\frac{2 \pi \rho_{B S} P_{t x} A}{\eta-2}\left[\left(2 R_{c}-r_{u}\right)^{2-\eta}-\left(R_{n w}-r_{u}\right)^{2-\eta}\right] .
\end{aligned}
$$

Hence, replacing $p_{u, b}=P_{t x} A r_{u}^{-\eta}$, together with $p_{u, C o M P}$ of Eq. (6) and $p_{u, e x t}$ of Eq. (7), we can compute $\Gamma_{u}^{C o M P}$ in Eq. (4). Furthermore, according to Shannon's formula, the maximum theoretical achievable data rate $D_{u}(r)$ can be computed as $D_{u}(r)=B_{u} \times \log _{2}\left(1+\Gamma_{u}^{C o M P}(r)\right)$, where $B_{u}$ is the UE's bandwidth. Hence, the total data rate $D_{\text {area }}$ over a network area of radius $R_{a}$, can be computed as:

$$
D_{\text {area }}=\frac{B \pi}{\sqrt{3} R_{c}^{2}} \int_{0}^{R_{a}} r \log _{2}\left(1+\Gamma_{u}^{C o M P}(r)\right) d r .
$$

When $R_{a}=R_{e}$, the above equation is evolved to compute the total cell data rate, $D_{\text {cell }}^{C o M P}$.

\section{Simulation AND RESUlts}

Several purposes are exposed in this section as follows. First, we show some numerical results of the data rate $D_{\text {area }}$ over both macro cellular network (denoted MCN) and femto cellular network (denoted FCN). Then, we present the accuracy of the EE expressions proposed in the last section by comparing the simulation results to those of Monte Carlo (MC) simulations of a hexagonal network. Finally, we show the impact of the number of coordinated BSs, $n$, on energy efficiency improvement and investigate the variation of the backhauling power consumption.

For MC simulations, we consider 7 rings of hexagonal cells around a central hexagon such that $R_{n w}=15 R_{c} . N_{u}$ UEs are generated uniformly in the central hexagon and we assume

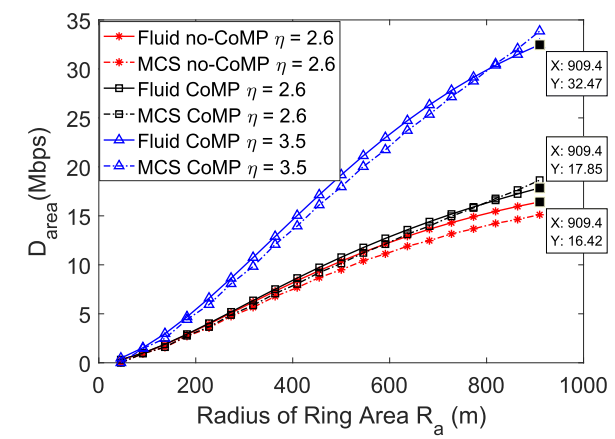

Fig. 3: $D_{\text {area }}$ vs radius of the MCN, $R=1000 \mathrm{~m}, K_{C o M P}=50 \mathrm{~W}$, $n=1$

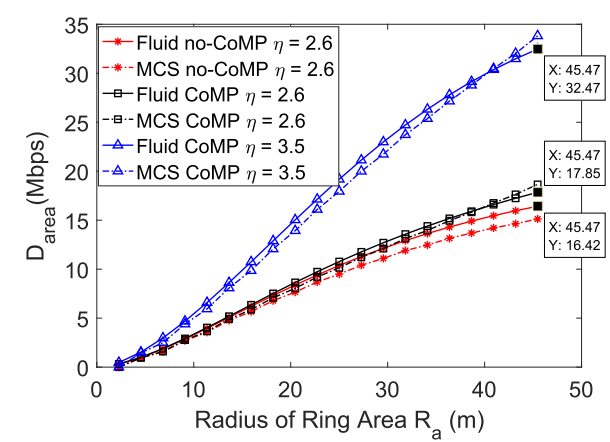

Fig. 4: $D_{\text {area }}$ vs radius of the FCN, $R=50 m, K_{C o M P}=30 m W, n=1$

that they are attached to the BS located at the center of the hexagon. We compute the $\Gamma_{u}$ (without JT-CoMP case), $\Gamma_{u}^{C o M P}$ (in case of JT-CoMP), for each UE in the area and then sum the achievable data rate for all the UEs depending on $D_{u}=B_{u} \times \log _{2}\left(1+\Gamma_{u}\right)$ or $D_{u}=B_{u} \times \log _{2}\left(1+\Gamma_{u}^{C o M P}\right)$, related to two cases above respectively. Finally we obtain the total data rate $D_{\text {area }}$ in the area. The results presented here are obtained by averaging over 5000 independent iterations of MC simulations. The other simulation parameters are set up according to Table I for the power consumption model as defined in [16], and Table II for the other network parameters.

Fig. 3 and Fig. 4 depict the data rate $D_{\text {area }}$ as a function of the network radius $R_{a}$ for two path-loss exponent $\eta=2.6$ or $\eta=3.5$ in the MCN and FCN, respectively. The results confirm that the proposed model is effective and match well with MC results for the both cases of JT-CoMP and without JT-CoMP, whatever the type of the cellular network and the values of $\eta$. Moreover, the results in both figures show that the theoretical $D_{\text {area }}$ increases with the size of the network area $R_{a}$ when the JT-CoMP is applied. Indeed, the data rate over the network area is related to $R_{a}$, as defined in Eq. (8). Alternatively, while comparing Fig. 3 and Fig. 4, we observe that the numerical values of $D_{\text {area }}$ are identical. For example, for $R_{a}=R_{e}$ (the whole cell) and $\eta=2.6$, the data rate is constant $D_{\text {area }}=17.85 \mathrm{Mbps}$. Therefore, the data rate enhancement is the same, regardless of the type of the cellular network. In fact, if we introduce the normalized distance $x=r_{u} / R_{c}$, and considering $R_{n w}=15 R_{c}, \Gamma_{u}^{C o M P}\left(r_{u}\right)$ can 


$$
\Gamma_{u}^{C o M P}(x)=\frac{x^{-\eta}+\frac{n \pi}{6 \sqrt{3}(\eta-2)}\left[(2-x)^{2-\eta}-(4-x)^{2-\eta}\right]}{\left.\frac{\pi}{\sqrt{3}(\eta-2)}\left[(2-x)^{2-\eta}-(15-x)^{2-\eta}\right]\right]+\frac{n \pi}{6 \sqrt{3}(\eta-2)}\left[(2-x)^{2-\eta}-(4-x)^{2-\eta}\right]}
$$

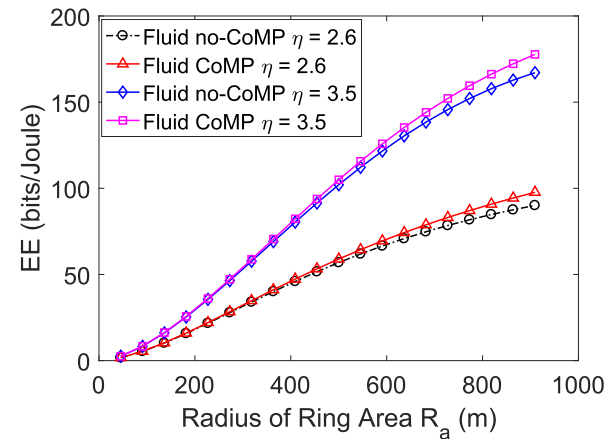

Fig. 5: EE vs radius of the $\mathrm{MCN}, R=1000 \mathrm{~m}, K_{C o M P}=50 \mathrm{~W}, n=1$

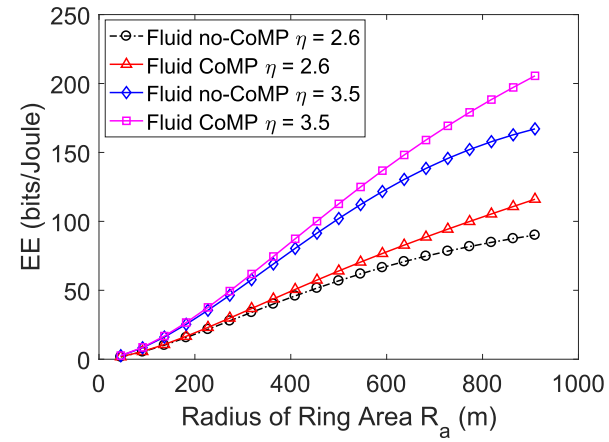

Fig. 6: EE vs radius of the $\mathrm{MCN}, R=1000 \mathrm{~m}, K_{C o M P}=50 \mathrm{~W}, n=3$

be rewritten as Eq. (9).

With $R_{a}=k R_{c}$, then the total data rate $D_{\text {area }}$ over a network area of radius of $R_{a}$ can be rewritten as:

$$
\begin{aligned}
D_{\text {area }} & =\frac{B \pi}{\sqrt{3} R_{c}^{2}} \int_{0}^{R_{a}} r \log _{2}\left(1+\Gamma_{u}^{C o M P}(r)\right) d r \\
& =\frac{B \pi}{\sqrt{3}} \int_{0}^{k} x \log _{2}\left(1+\Gamma_{u}^{C o M P}(x)\right) d x
\end{aligned}
$$

As a result, $D_{\text {area }}$ does not depend on $R_{c}$ and $R$, but is related to the ratios of $R_{a} / R_{c}, R_{n w} / R_{c}$ and $\eta$. In other words, we can obtain same data rate in the MCN and the FCN, if the same path-loss exponent and the same bandwidth are set together with the same distance ratios of $R_{a} / R_{c}$ and $R_{n w} / R_{c}$.

Fig. 5 and Fig. 6 depict the EE performance as a function of various $R_{a}$ values in a MCN. Here, we consider two pathloss exponents $\eta=2.6,3.5$ and $n=1,3$, the number of coordinated BSs. In a FCN, the results are shown in Fig. 7 and Fig. 8. All these results are obtained in case of a constant backhauling power cost, i.e. $K_{C o M P}=50 \mathrm{~W}$ for a $\mathrm{MCN}$ and $K_{C o M P}=30 \mathrm{~mW}$ for a FCN as in [17]. We observe that the numerical results of EE are improved with JT-CoMP scheme compared to those without JT-CoMP, in both networks. Especially, while comparing the numerical values in Fig. 5 and

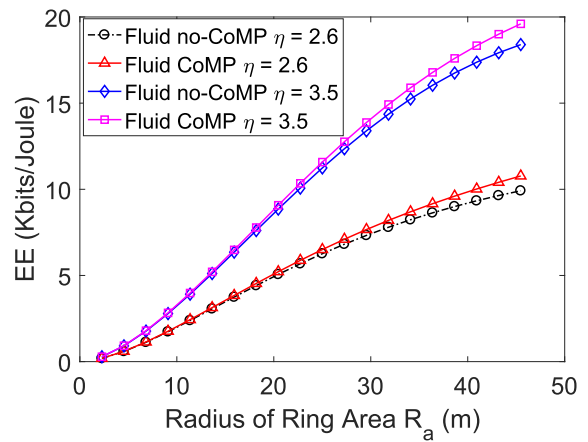

Fig. 7: EE vs radius of the FCN, $R=50 m, K_{C o M P}=30 m W, n=1$

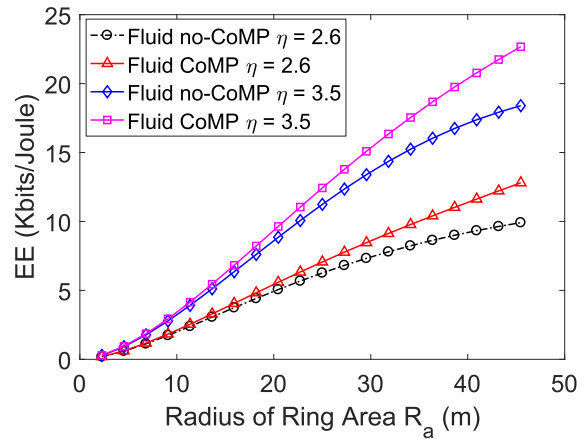

Fig. 8: EE vs radius of the FCN, $R=50 m, K_{C o M P}=30 m W, n=3$

Fig. 6 for $\eta=3.5$, we observe that the EE improvement is more important when 3 coordinated BSs are used than the case where only one BS is considered $(n=1)$. Increasing the number of cooperating BSs improves the data rate over the network area in the case of fixed backhauling power consumption. The same conclusion is found when comparing the numerical results related to the FCN case in Fig. 7 and Fig. 8.

Furthermore, all these figures show that the EE improvement is significant when $R / 2<R_{a}<R_{e}$, whatever the type of the cellular network. Indeed, JT-CoMP is not appropriate for near UEs, i.e. UEs closer to their serving BS since they experience a great signal quality. Therefore, it is interesting to analyze the impact of $n$, the number of coordinated BSs on the EE performance while comparing with the baseline case where no joint transmission is applied (no-CoMP) for far UEs, located at distance larger than $R / 2$.

Fig. 9 and Fig. 10 show the numerical values of EE for various number of coordinated BSs $n$ and $\eta=2.6$ for $R / 2<R_{a}<R_{e}$, in a $\mathrm{MCN}$ and a $\mathrm{FCN}$, respectively. In both figures, we observe that EE is improved as the growth of $n$ for a fixed $R_{a}$. In fact, for a fixed $R_{a}$, the data rate $D_{\text {area }}$ increases with the raise of coordinated BSs number 


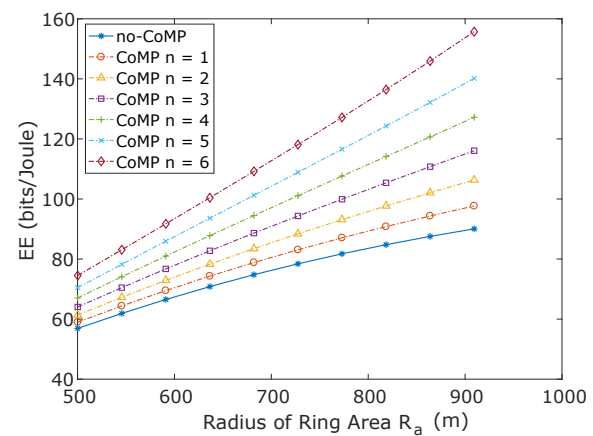

Fig. 9: EE improvement in a MCN, $R=1000 m, \eta=2.6, K_{C o M P}=50 \mathrm{~W}$

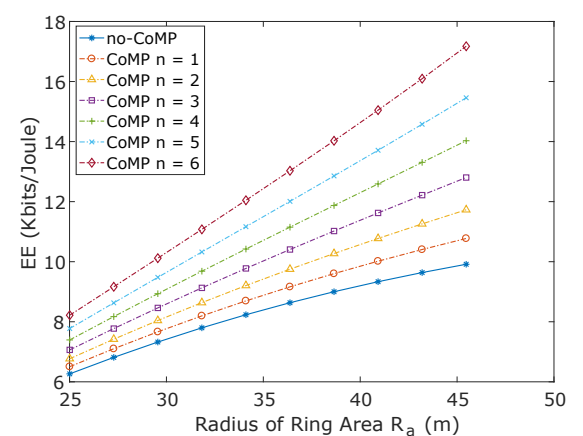

Fig. 10: EE improvement in a FCN, $R=50 m, \eta=2.6, K_{C o M P}=$ $30 m W$

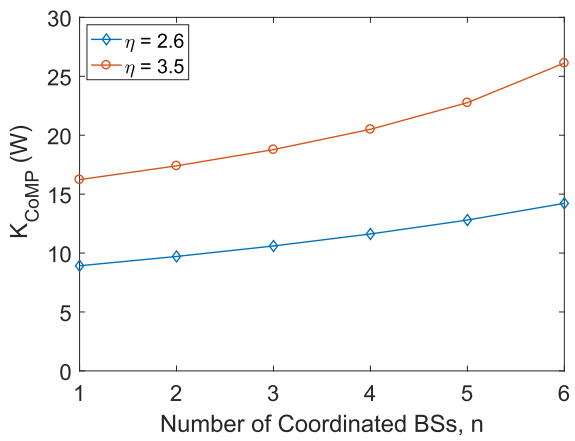

Fig. 11: $K_{C o M P}$ vs the number of coordinated BSs $n$ in a MCN

$n$, regarding the fixed backhauling power cost. In the MCN, the EE enhancement reaches 66bits/Joule when 6 BSs are used to jointly transmit data, against 26bits/Joule in case of 3 BSs. The EE improvement is more substantial in a FCN, since it is about 2.9 Kbits/Joule when 3 BSs are considered and reaches $7.3 \mathrm{Kbits} /$ Joule in case of 6 coordinated BSs.

Additionally, comparing Fig. 6 and Fig. 8 for $\eta=3.5$ and $n=3$, we observe that EE is improved about 38bits/Joule at the edge of a MCN, whereas it is about $4.3 \mathrm{Kbits} /$ Joule in a FCN. This means that the JT-CoMP is more effective in small cellular networks and bring high improvement than macrocell. Although the same data rate $D_{\text {area }}$ is observed in the $\mathrm{MCN}$ and the FCN, the EE improvement is higher in the FCN than in MCN, thanks to the smaller fixed backhauling power cost in the FCN.

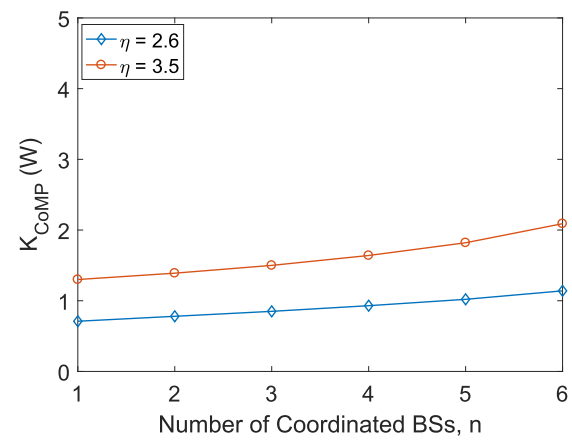

Fig. 12: $K_{C o M P}$ vs the number of coordinated BSs $n$ in a FCN

TABLE III: Numerical Results of $E E_{\text {cell }}$ measured by Kbits/Joule in the FCN for fixed $K_{C o M P}$ and various $K_{C o M P}$ with $\eta=2.6$

\begin{tabular}{|c|c|c|c|c|c|c|c|}
\hline & \multirow{2}{*}{$\begin{array}{l}\text { no- } \\
\text { CoMP }\end{array}$} & \multicolumn{6}{|c|}{ CoMP Mode } \\
\hline & & $\begin{array}{l}n= \\
1\end{array}$ & $\begin{array}{l}n= \\
2\end{array}$ & $\begin{array}{l}n= \\
3\end{array}$ & $\begin{array}{l}n= \\
4\end{array}$ & $\begin{array}{l}n= \\
5\end{array}$ & $\begin{array}{l}n= \\
6\end{array}$ \\
\hline $\begin{array}{l}E E_{\text {cell }} \\
\text { (Fixed } \\
K_{C o M P} \text { ) }\end{array}$ & 9.91 & 10.77 & 11.73 & 12.8 & 14.03 & 15.46 & 17.17 \\
\hline $\begin{array}{l}E E_{\text {cell }} \\
\text { (Various } \\
K_{\text {CoMP }} \text { ) }\end{array}$ & 9.91 & 10.74 & 11.69 & 12.76 & 13.98 & 15.4 & 17.1 \\
\hline
\end{tabular}

However, when a new cooperative $\mathrm{BS}$ is added in the system, the additional energy needs are larger due to the additional power consumption for transmitting the backhauling traffic. Therefore, based on Eq. (3), Fig. 11 and Fig. 12 show some numerical results of the backhauling power consumption, $K_{C o M P}$, along with the various number of coordinated BSs $n$ in a MCN and a FCN, for $\eta=2.6$ and 3.5. We observe that the values of $K_{C o M P}$ in a femtocell network increases slightly with the raise of $n$ whatever the values of $\eta$. Regarding the macrocell case, the additional energy cost for transmitting the backhauling capacity between BSs while adding the new coordinated BS is more significant. Specially, the results show that $K_{C o M P}$ is about $26 W$ for a MCN in Fig. 11 for $\eta=3.5$ and $n=6$, and it is about $2 W$ for a FCN in Fig. 12. Since the FCN has lower power dissipation compared to the MCN, which causes the smaller $K_{C o M P}$ in FCN.

For convenient presentation purpose, we compare in Table. III, the numerical values of EE per cell (denoted as $E E_{\text {cell }}$ when $R_{a}=R_{e}$ ) obtained from fluid model in a FCN regarding of the two cases: a constant backhauling power cost $K_{C o M P}=30 \mathrm{~mW}$ and variable $K_{C o M P}$, calculated by Eq. (3). As presented previously, we observe that $E E_{\text {cell }}$ increases with the growth of $n$ in the two cases, which is due to the higher data rate improvement brought by JT-CoMP. Moreover, the results show that $E E_{\text {cell }}$ gain is about $1.8 \mathrm{Kbits} / J$ oule when there are 2 coordinated BSs in the first ring. $E E_{\text {cell }}$ gain is around $2.8 \mathrm{Kbits} /$ Joule when 3 coordinated BSs are utilized regardless of the cases of $K_{C o M P}$. Moreover, it is to emphasize that the EE over the cell are quite similar in both cases. Therefore, considering $K_{C o M P}=30 \mathrm{~mW}$ is reasonable choice, it does not compromise the first results presented above. The same observations are verified (Table. IV in case 
TABLE IV: Numerical Results of $E E_{\text {cell }}$ measured by bits/Joule in the MCN for fixed $K_{C o M P}$ and various $K_{C o M P}$ with $\eta=2.6$

\begin{tabular}{|c|c|c|c|c|c|c|c|}
\hline & \multirow{2}{*}{$\begin{array}{l}\text { no- } \\
\text { CoMP }\end{array}$} & \multicolumn{6}{|c|}{ CoMP Mode } \\
\hline & & $\begin{array}{l}n= \\
1\end{array}$ & $\begin{array}{l}n= \\
2\end{array}$ & $\begin{array}{l}n= \\
3\end{array}$ & $\begin{array}{l}n= \\
4\end{array}$ & $\begin{array}{l}n= \\
5\end{array}$ & $\begin{array}{l}n= \\
6\end{array}$ \\
\hline $\begin{array}{l}E E_{\text {cell }} \\
\text { (Fixed } \\
\left.K_{C o M P}\right)\end{array}$ & 90.05 & 97.68 & 106.34 & 116.07 & 127.19 & 140.15 & 155.71 \\
\hline $\begin{array}{l}E E_{\text {cell }} \\
\text { (Various } \\
K_{C o M P} \text { ) }\end{array}$ & 90.05 & 97.83 & 106.5 & 116.25 & 127.38 & 140.35 & 155.92 \\
\hline
\end{tabular}

of a MCN with $K_{C o M P}=50 \mathrm{~W}$ ).

In conclusion, the obtained results emphasize the JT-CoMP scheme in small cells as opposed to macrocells. It is a very interesting result that matches very well with the future $5 \mathrm{G}$ scenarios characterized by high density and more short-range communication such as IoT.

\section{CONCLUSION}

In this paper, we proposed a tractable expression of the energy efficiency (EE) performance based on fluid modeling of the downlink transmission system while considering the JTCoMP approach. Then, we investigated the EE enhancement in both types of cellular networks: macro $(\mathrm{MCN})$ and femto (FCN), through a comparison with the baseline case where no coordination is applied. The numerical results show the model accuracy of EE through a comparison with Monte Carlo trials. Moreover, the results exhibit that the data rate is the same in the both types of cellular networks, whereas the energy efficiency in a FCN is larger than the ones in a MCN. In both networks, the EE is improved with the raise of the number of coordinated BSs $n$ for the two cases: fixed backhauling power cost $K_{C o M P}$ and variable $K_{C o M P}$, since the total data rate of the area increases when $n$ increases. Furthermore, for variable $K_{C o M P}$, the backhauling power cost also increases with the growth of $n$, due to the additional energy cost for transmitting the additional backhauling capacity between macro BSs. However, in the FCN, adding a new BS in the coordination set, produces a slight change in the backhauling power cost $K_{C o M P}$. Consequently, JT-CoMP is more efficient in the small cellular network, which explains that small cell deployments are more utilized in future $5 \mathrm{G}$ networks and fit with the main $5 \mathrm{G}$ scenarios characterized by high nodes density and short range transmissions.

In the next step, we intend to use this proposed framework of EE to investigate the signal quality (SIR) threshold, since JT-CoMP can not bring obvious improvement for UEs, which are close to their serving BS.

\section{REFERENCES}

[1] I. Vision, "Framework and overall objectives of the future development of IMT for 2020 and beyond," International Telecommunication Union (ITU), Document, Radiocommunication Study Groups, 2015.

[2] "5G public private partnership 5G manifesto," Mobile World Congress, March 2015.

[3] M. De Sanctis, E. Cianca et al., "Energy efficient wireless networks towards green communications," Wireless Personal Communications, vol. 59, no. 3, pp. 537-552, 2011.
[4] D. Pompili, A. Hajisami et al., "Elastic resource utilization framework for high capacity and energy efficiency in cloud RAN," IEEE Communications Magazine, vol. 54, no. 1, pp. 26-32, 2016.

[5] E. Björnson, L. Sanguinetti et al., "Optimal design of energy-efficient multi-user MIMO systems: Is massive MIMO the answer?" IEEE Transactions on Wireless Communications, vol. 14, no. 6, pp. 30593075, 2015.

[6] K. S. V. Prasad, E. Hossain et al., "Energy efficiency in Massive MIMOBased 5G networks: Opportunities and challenges," IEEE Wireless Communications, 2017.

[7] Q. Cui, T. Yuan et al., "Energy-efficient two-way relaying under nonideal power amplifiers," IEEE Transactions on Vehicular Technology, vol. 66, no. 2, pp. 1257-1270, 2017.

[8] S. Buzzi, I. Chih-Lin et al., "A survey of energy-efficient techniques for $5 \mathrm{G}$ networks and challenges ahead," IEEE Journal on Selected Areas in Communications, vol. 34, no. 4, pp. 697-709, 2016.

[9] S.-Y. Kim and C.-H. Cho, "Call blocking probability and effective throughput for call admission control of CoMP joint transmission," IEEE Transactions on Vehicular Technology, vol. 66, no. 1, pp. 622-634, 2017.

[10] J.-M. Kelif, M. Coupechoux et al., "A fluid model for performance analysis in cellular networks," EURASIP Journal on Wireless Coтmunications and Networking, vol. 2010, no. 1, p. 435189, 2010.

[11] L. Zitoune, S. Cerovic et al., "Performance evaluation of JT CoMP approach: Tractable model using spatial fluid modeling," IFIP Networking Conference (IFIP Networking '16), pp. 198-206, 2016.

[12] Y. Hou, L. Zitoune et al., "Fluid modeling of energy efficiency in large cellular networks," IEEE 29th Annual International Symposium on Personal, Indoor and Mobile Radio Communications (PIMRC '18), pp. 1-5, 2018.

[13] T. Zhang, J. Zhao et al., "Energy efficiency of base station deployment in ultra dense HetNets: A stochastic geometry analysis," IEEE Wireless Communications Letters, vol. 5, no. 2, pp. 184-187, 2016.

[14] A. J. Fehske, P. Marsch et al., "Bit per joule efficiency of cooperating base stations in cellular networks," IEEE Globecom Workshops (GCWkshps '10), pp. 1406-1411, 2010.

[15] D. Liu and C. Yang, "Energy efficiency of downlink networks with caching at base stations," IEEE Journal on Selected Areas in Communications, vol. 34, no. 4, pp. 907-922, 2016.

[16] F. Héliot, M. A. Imran et al., "On the energy efficiency-spectral efficiency trade-off over the MIMO Rayleigh fading channel," IEEE Transactions on Communications, vol. 60, no. 5, pp. 1345-1356, 2012.

[17] B. Du, C. Pan et al., "Distributed energy-efficient power optimization for CoMP systems with max-min fairness," IEEE Communications Letters, vol. 18, no. 6, pp. 999-1002, 2014. 